Ferrata Storti Foundation

\title{
Health-related quality of life in transplant ineligible newly diagnosed multiple myeloma patients treated with either thalidomide or lenalidomide-based regimen until progression: a prospective, open-label, multicenter, randomized, phase 3 study
}

Haematologica 2020

Volume 105(1):1650-1659

\section{Correspondence:}

LENE KONGSGAARD NIELSEN

lene.kongsgaard.nielsen@rsyd.dk

Received: April 19, 2019.

Accepted: September 11, 2019.

Pre-published: September 12, 2019.

doi:10.3324/haematol.2019.222299

Check the online version for the most updated information on this article, online supplements, and information on authorship \& disclosures: www. haematologica.org/content/105/6/1650

(C)2020 Ferrata Storti Foundation

Material published in Haematologica is covered by copyright. All rights are reserved to the Ferrata Storti Foundation. Use of published material is allowed under the following terms and conditions:

https://creativecommons.org/licenses/by-nc/4.0/legalcode. Copies of published material are allowed for personal or internal use. Sharing published material for non-commercial purposes is subject to the following conditions:

https://creativecommons.org/licenses/by-nc/4.0/legalcode, sect. 3. Reproducing and sharing published material for commercial purposes is not allowed without permission in writing from the publisher.

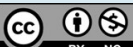

Lene Kongsgaard Nielsen, ${ }^{1 *}$ Claudia Stege, ${ }^{2 *}$ Birgit Lissenberg-Witte, ${ }^{3}$ Bronno van der Holt, ${ }^{4}$ Ulf-Henrik Mellqvist, ${ }^{5}$ Morten Salomo, ${ }^{6}$ Gerard Bos, ${ }^{7}$ Mark-David Levin, ${ }^{8}$ Heleen Visser-Wisselaar, ${ }^{4}$ Markus Hansson, ${ }^{9}$ Annette van der Velden, ${ }^{10}$ Wendy Deenik, ${ }^{11}$ Juleon Coenen, ${ }^{12}$ Maja Hinge, ${ }^{13}$ Saskia Klein, ${ }^{14}$ Bea Tanis, ${ }^{15}$ Damian Szatkowski, ${ }^{16}$ Rolf Brouwer, ${ }^{17}$ Matthijs Westerman, ${ }^{18}$ Rineke Leys, ${ }^{19}$ Harm Sinnige, ${ }^{20}$ Einar Haukås, ${ }^{21}$ Klaas van der Hem, ${ }^{22}$ Marc Durian, ${ }^{23}$ Peter Gimsing, ${ }^{24}$ Niels van de Donk, ${ }^{2}$ Pieter Sonneveld, ${ }^{25}$ Anders Waage, ${ }^{26}$ Niels Abildgaard $^{1}$ and Sonja Zweegman ${ }^{2}$

${ }^{1}$ Quality of Life Research Center, Department of Haematology, Odense University Hospital, Odense, Denmark; ${ }^{2}$ Amsterdam UMC, Vrije Universiteit Amsterdam, Department of Hematology, Cancer Center Amsterdam, Amsterdam, the Netherlands; ${ }^{3}$ Amsterdam UMC, Vrije Universiteit Amsterdam, Department of Epidemiology and Biostatistics, Amsterdam, the Netherlands; ${ }^{4} \mathrm{HOVON}$ Data Center, Department of Hematology, Erasmus MC Cancer Institute, Rotterdam, the Netherlands; 5 Section of Hematology and Coagulation, Department of Medicine, Sahlgrenska University Hospital, Gotheborg, Sweden; ${ }^{6}$ Department of Haematology, Rigshospitalet, Copenhagen, Denmark; ${ }^{7}$ Department of Haematology, Maastricht University Medical Center, Maastricht, the Netherlands;

${ }^{8}$ Department of Internal Medicine, Albert Schweitzer Hospital, Dordrecht, the Netherlands; ${ }^{9}$ Department of Haematology and Wallenberg Center for Molecular Medicine, Skåne University Hospital, Lund University, Lund, Sweden; ${ }^{10}$ Department of Internal Medicine, Martini Ziekenhuis, Groningen, the Netherlands; ${ }^{11}$ Department of Internal Medicine, Tergooi Ziekenhuis, Hilversum, the Netherlands; ${ }^{12}$ Department of Internal Medicine, Isala, Zwolle, the Netherlands; ${ }^{13}$ Department of Internal Medicine, Division of Hematology, Vejle Hospital, Vejle, Denmark; ${ }^{14}$ Department of Internal Medicine, Meander Medisch Centrum, Amersfoort, the Netherlands; ${ }^{15}$ Department of Internal Medicine, Groene Hart Ziekenhuis, Gouda, the Netherlands; ${ }^{16}$ Department of Oncology, Haematology and Palliative Care, Førde Central Hospital, Førde, Norway; ${ }^{17}$ Department of Internal Medicine, Reinier de Graaf Ziekenhuis, Delft, the Netherlands; ${ }^{18}$ Department of Internal Medicine, Northwest Clinics, Alkmaar, the Netherlands; ${ }^{19}$ Department of Internal Medicine, Maasstad Ziekenhuis, Rotterdam, the Netherlands; ${ }^{20}$ Department of Internal Medicine, Jeroen Bosch Ziekenhuis, Den Bosch, the Netherlands; ${ }^{21}$ Department of Haematology, Stavanger University Hospital, Stavanger, Norway, ${ }^{22}$ Department of Internal Medicine, Zaans Medisch Centrum, Zaandam, the Netherlands; ${ }^{23}$ Department of Internal Medicine, Tweesteden Ziekenhuis, Tilburg, the Netherlands; ${ }^{24}$ Department of Haematology, Rigshospitalet, Copenhagen, Denmark; ${ }^{25}$ Department of Haematology, Erasmus Medical Center Cancer Center, Rotterdam, the Netherlands and ${ }^{26}$ Department of Haematology, St Olavs Hospital and Norwegian University of Science and Technology, Trondheim, Norway

*LKN and CS contributed equally as co-first authors.

\section{ABSTRACT}

$\mathrm{D}$ ata on the impact of long term treatment with immunomodulatory drugs (IMiD) on health-related quality of life (HRQoL) is limited. The HOVON-87/NMSG18 study was a randomized, phase 3 study in newly diagnosed transplant ineligible patients with multiple myeloma, comparing melphalan-prednisolone in combination with thalidomide or lenalidomide, followed by maintenance therapy until progression (MPT-T or MPR-R). The EORTC OLO-C30 and MY20 questionnaires were completed at baseline, after three and nine induction cycles and six and 12 months of maintenance therapy. Linear mixed models and minimal important differences were used for evaluation. 596 patients participated in HRQoL reporting. Patients reported clinically relevant improvement in 
global quality of life (QoL), future perspective and role and emotional functioning, and less fatigue and pain in both arms. The latter being of large effect size. In general, improvement occurred after 6-12 months of maintenance only and was independent of the World Health Organisation performance at baseline. Patients treated with MPR-R reported clinically relevant worsening of diarrhea, and patients treated with MPT-T reported a higher incidence of neuropathy. Patients who remained on lenalidomide maintenance therapy for at least three months reported clinically meaningful improvement in global QoL and role functioning at six months, remaining stable thereafter. There were no clinically meaningful deteriorations, but patients on thalidomide reported clinically relevant worsening in neuropathy. In general, HRQoL improves both during induction and maintenance therapy with immunomodulatory drugs. The side effect profile of treatment did not negatively affect global QoL, but it was, however, clinically relevant for the patients. (Clinicaltrials.gov identifier: NTR1630).

\section{Introduction}

Multiple myeloma (MM) is a malignancy of the plasma cells in the bone marrow. Patients with $\mathrm{MM}$ are at high risk of developing bone destructions and fractures, hypercalcaemia, renal failure and anemia. ${ }^{1,2}$ Compared to patients with other hematological malignancies, patients with $\mathrm{MM}$ report a higher incidence and severity of symptoms with a reduced health-related quality of life (HRQoL) as a consequence..$^{3-5}$ However, there is limited data on the effect of first line treatment on HRQoL in transplant ineligible, newly diagnosed patients with $\mathrm{MM}$ (NDMM), especially throughout maintenance treatment. ${ }^{6}$ In several of these trials, the immunomodulatory drugs (IMiDs) thalidomide and/or lenalidomide were investigated. ${ }^{7-13}$ HRQoL during treatment with thalidomide and lenalidomide were compared head to head in the FIRST and the ECOG E1A06 trials only. ${ }^{10,14}$

The FIRST trial compared continuous therapy with lenalidomide and dexamethasone (Rd), with Rd for 18 months, and melphalan-prednisone-thalidomide (MPT) for 18 months. ${ }^{14}$ Clinically relevant changes were published only for six HRQoL scales. ${ }^{9}$ These were preselected as they were perceived to be clinically relevant. Both $\mathrm{Rd}$ and MPT resulted in a statistically significant improvement in all subscales, except side effects of treatment that worsened over time in both arms. There were no differences between arms in global quality of life (QoL), physical functioning, pain and fatigue; although $\mathrm{Rd}$ treated patients reported significantly less side effects of treatment and less disease symptoms at three months compared to MPT. A post hoc prediction model was developed, suggesting that HRQoL was at least maintained or further improved beyond 18 months. Unfortunately, the effect of $\mathrm{Rd}$ continuous versus 18 months only on HRQoL cannot be deduced with certainty from this study, as HRQoL data beyond 18 months was lacking. ${ }^{13}$

In contrast to the FIRST trial, in the ECOG E1A06 trial, the Functional Assessment of Cancer TherapyNeurotoxicity Trial Outcome Index (FACT-Ntx TOI) score was used for HRQoL evaluation, instead of the European Organisation for Research and Treatment of Cancer (EORTC) OLO-C30. It was shown that melphalan-prednisone-lenalidomide (MPR) followed by lenalidomide maintenance (MPR-R) resulted in a superior HRQoL after 12 months only, compared to MPT followed by thalidomide maintenance (MPT-T). ${ }^{10}$ However, the HRQoL effects of lenalidomide and thalidomide maintenance therapies were not investigated separately.
The effect of MPR-R on HRQoL might be deduced from the MM-015 trial, comparing MPR-R, MPR and melphalan-prednisolone (MP). ${ }^{11,15}$ After six months of maintenance, patients who actually received MPR-R therapy reported a statistically significant improvement in HRQoL in 5 of 6 subscales, versus in 2 of 6 subscales only in MP and MPR treated patients. However, also from this study, HRQoL data beyond six months of maintenance therapy is lacking. ${ }^{6,11}$

Recently, the data from the MRC IX study showed that maintenance therapy with thalidomide resulted in an inferior global QoL after three months with a persistent trend for detriment at six and 12 months. ${ }^{12}$ This is important as, especially after achieving disease control with induction therapy, long-term continuation of maintenance therapy may turn the pros of maintenance therapy into cons because of side effects that negatively affect HRQoL. The data on lenalidomide maintenance therapy, however, is limited..$^{10,11,13,16}$ Data from a prospective observational cohort study showed no negative impact of lenalidomide maintenance therapy following autologous stem cell transplantation. ${ }^{16}$ To the best of our knowledge, prospective analyses after six months of maintenance therapy in non-transplant eligible patients with NDMM are lacking. Only the post hoc analysis of the FIRST trial is available. ${ }^{13}$

We here report data on all the collected HRQoL subscales from the open-label, randomized HOVON87/NMSG18 study, thereby providing HRQoL data, not only during induction, but also during maintenance therapy with lenalidomide and thalidomide therapy. ${ }^{17}$ Although currently both IMiD-based regimens are mainly replaced by $\mathrm{Rd}$ continuously, either combined with bortezomib during induction or not, the impact of long term lenalidomide on HRQoL is of interest. In addition, we discuss methods to account for the impact of differences in discontinuation rate due to toxicity between the two arms on the outcome of HRQoL analysis.

\section{Methods}

\section{Study design}

Study details have been published previously. ${ }^{17}$ In brief, symptomatic patients with NDMM $>65$ years of age or transplant ineligible patients $\leq 65$ years were included. Patients were randomized between nine 28-day induction cycles of MPT, followed by thalidomide maintenance (MPT-T) or nine 28-day induction cycles of MPR followed by lenalidomide maintenance 
(MPR-R). Maintenance treatment was given until progression, intolerable side effects or other conditions that required treatment discontinuation. The study protocol was approved by the Ethics Committee, and written informed consent was obtained from all participants. The study was registered at $w w w$.trialregister.nl as NTR1630.

\section{Health-related quality of life assessments}

Participation in the HRQoL reporting was optional. Questionnaires were given to the patients at baseline (T0), after induction cycle 3 (T1) and cycle 9 (T2), and after six (T3) and 12 (T4) months of maintenance therapy.

For HRQoL assessment, two EORTC QoL questionnaires were used; the OLQ-C30 and the Myeloma specific OLOMY20. ${ }^{18,19}$ The OLQ-C30 contains five functional scales, nine symptom scales and one global QoL scale. ${ }^{20,21}$ The OLQ-MY20 contains two functional and two symptom scales. For the evaluation of peripheral neuropathy, question 13 of the QLQ-MY20 "Did you have tingling hands or feet?" was used. The EORTC manual $^{21}$ was used to calculate all HRQoL scales. A detailed description of the questionnaires and neuropathy scale, data collection and assignment of the questionnaires to T0-T4 is found in the Online Supplementary Material and Methods.

\section{Statistical analyses}

Change in HRQoL over time was assessed by linear mixed models, both "within arms" and "between arms", from T0 to T4, as well as from T2 to T4 for patients who had at least three months of maintenance therapy. A $P$-value $<0.005$ was considered statistically significant as multiple subscales were tested. Model estimates were used for post hoc comparisons of changes from baseline. ${ }^{21}$ A change in mean HRQoL score was defined as clinically meaningful if it was above the minimal important difference (MID) threshold using distribution-based MID calculated for both QLQ-C30 and MY20 subscales. ${ }^{22}$ MID between arms was defined as $>5$ points difference at a specific time point. ${ }^{22}$ For QLO-C30 subscales, an additional anchor-based method by Cocks was used, assessing whether HRQoL changes and differences were of small, medium or large effect. ${ }^{23,24}$ Details are described in the Online Supplementary Material and Methods.

To check for effect modification by the World Health Organisation (WHO) performance, sex, age and treatment response, linear mixed models included fixed effects for time, WHO, sex, age, treatment response and their two-way interaction and a random slope for subject.

HRQoL questionnaires were not systematically collected from patients who discontinued treatment, which might introduce a bias when comparing HRQoL. Therefore, we investigated the impact of missing data due to treatment discontinuation on changes in HRQoL over time. We compared HRQoL of i) patients on and off protocol matched by timing, ii) patients who discontinued therapy before or after start of maintenance, and iii) patients who discontinued therapy because of peripheral neuropathy versus patients still on protocol until 12 months maintenance therapy. For a detailed description, see the Online Supplementary Appendix Materials and Methods.

For statistical analysis, SPSS version 22.0 was used.

\section{Results}

Ninety-four percent (596) of the 637 patients included in the HOVON-87/NSMG18 trial gave informed consent for participation in the HRQoL study. Only patients who filled out a baseline questionnaire were included in the
Table 1. Demographic characteristics of the patients included in the health-related quality of life analysis.

\begin{tabular}{|c|c|c|}
\hline Demographic characteristics & $\begin{array}{l}\text { MPTT } \\
(\mathrm{N}=272)\end{array}$ & $\begin{array}{c}\text { MPR-R } \\
(\mathrm{N}=281)\end{array}$ \\
\hline $\begin{array}{c}\text { Median age, years (IQR) } \\
\text { Age } \geq 76 \text { years, } N(\%)\end{array}$ & $\begin{array}{l}72(69-77) \\
90(33 \%)\end{array}$ & $\begin{array}{l}73(69-77) \\
98(35 \%)\end{array}$ \\
\hline $\begin{array}{l}\text { Sex, N (\%) } \\
\text { Male } \\
\text { Female }\end{array}$ & $\begin{array}{l}133(49 \%) \\
139(51 \%)\end{array}$ & $\begin{array}{l}164(58 \%) \\
117(42 \%)\end{array}$ \\
\hline $\begin{array}{l}\text { WHO performance, } \mathrm{N}(\%) \\
0 \\
1 \\
2 \\
3 \\
\text { Unknown }\end{array}$ & $\begin{array}{c}89(33 \%) \\
132(49 \%) \\
39(14 \%) \\
5(2 \%) \\
6(2 \%)\end{array}$ & $\begin{array}{c}107(38 \%) \\
124(44 \%) \\
40(14 \%) \\
5(2 \%) \\
5(2 \%)\end{array}$ \\
\hline $\begin{array}{l}\text { M-protein subtype, N (\%) } \\
\text { IgG } \\
\text { IgA } \\
\text { IgD } \\
\text { Light chain only } \\
\text { Unknown }\end{array}$ & $\begin{array}{c}177(65 \%) \\
73(27 \%) \\
4(2 \%) \\
17(6 \%) \\
0(0 \%)\end{array}$ & $\begin{array}{l}176(63 \%) \\
69(25 \%) \\
1(<0.5 \%) \\
34(12 \%) \\
1(<0.5 \%)\end{array}$ \\
\hline $\begin{array}{l}\text { ISS, N (\%) } \\
\text { I } \\
\text { II } \\
\text { III } \\
\text { Unknown/missing }\end{array}$ & $\begin{array}{c}61(23 \%) \\
134(49 \%) \\
74(27 \%) \\
3(1 \%)\end{array}$ & $\begin{array}{c}78(28 \%) \\
136(48 \%) \\
65(23 \%) \\
2(1 \%)\end{array}$ \\
\hline $\begin{array}{l}\text { Lytic bone lesions, } \mathrm{N}(\%) \\
\text { None } \\
1 \\
2 \\
3 \text { or more } \\
\text { Unknown/missing }\end{array}$ & $\begin{array}{c}86(32 \%) \\
25(9 \%) \\
15(6 \%) \\
141(52 \%) \\
5(2 \%)\end{array}$ & $\begin{array}{c}89(32 \%) \\
19(7 \%) \\
19(7 \%) \\
150(53 \%) \\
4(1 \%)\end{array}$ \\
\hline $\begin{array}{l}\text { FISH performed, N (\%) } \\
\text { Yes }\end{array}$ & $206(76 \%)$ & $220(78 \%)$ \\
\hline $\begin{array}{l}\text { FISH abnormality if performed, } N(\%) \\
17 p 13 \text { loss } \\
\mathrm{t}(4 ; 14)(\mathrm{p} 16 ; \mathrm{q} 32) \\
\mathrm{t}(14 ; 16)(\mathrm{q} 32 ; \mathrm{q} 23) \\
\text { lq21 gain }\end{array}$ & $\begin{array}{c}23 / 188(12 \%) \\
18 / 199(9 \%) \\
2 / 170(1 \%) \\
56 / 146(38 \%)\end{array}$ & $\begin{array}{l}16 / 196(8 \%) \\
17 / 216(8 \%) \\
10 / 192(5 \%) \\
58 / 165(35 \%)\end{array}$ \\
\hline
\end{tabular}

MPTT: melphalan-prednisone-thalidomide induction and thalidomide maintenance therapy; MPR-R: melphalan-prednisone-lenalidomide induction and lenalidomide maintenance therapy; n: number of patients; IQR: interquartile range, WHO: World Health Organisation; ISS: International Staging System; FISH: fluorescence in situ hybridization.

HRQoL analysis; 272 patients in MPT-T versus 281 patients in MPR-R. The patient- and disease characteristics of the HRQoL cohort (Table 1) were comparable to the original study population. ${ }^{17}$ The patient flow and drop-out during study are presented in the CONSORT diagram in Figure 1. Fewer patients in the MPT-T arm started maintenance compared to patients in the MPR-R arm, $146(54 \%)$ versus $174(62 \%)$. In addition, more patients discontinued MPT-T than MPR-R (first year discontinuation rate; $68 \%$ vs. $30 \% ; P<0.001)$. The main reason for discontinuation was peripheral neuropathy for thalidomide and hematological toxicity for lenalidomide. ${ }^{17}$ A graphic presentation of the number of patients on protocol and the number of completed questionnaires at each scheduled time point, ranging from $69-87 \%$, is presented in the Online Supplementary Figure S1. At baseline, no significant differences existed between the treatment arms (Online Supplementary Table S1). 


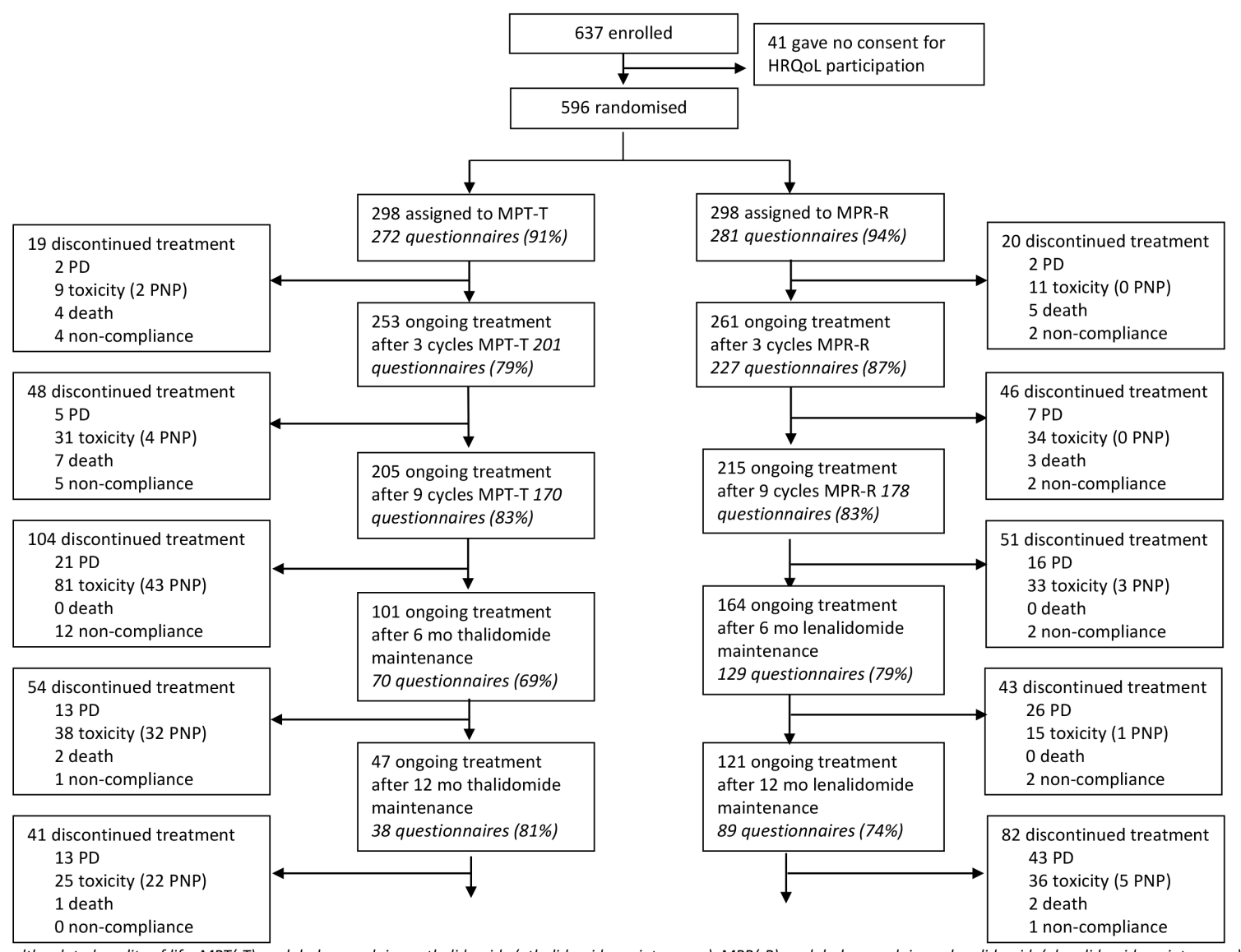

HRQOL: health-related quality of life; MPT(-T): melphalan-prednisone-thalidomide (+thalidomide maintenance); MPR(-R): melphalan-prednisone-lenalidomide(+lenalidomidemaintenance), $P D$ : progressive disease; PNP: peripheral neuropathy

Figure 1. Consort diagram. Consort diagram of the number of patients participating in the health-related quality of life (HRQoL) study, the number of answered questionnaires and the number of patients off protocol and reason for treatment discontinuation.

\section{Percentage of patients reaching a clinically meaningful change in HRQOL}

In both arms, clinically relevant improvement in global QoL was more prominent than deterioration (Figure 2). During MPT induction therapy, improvement occurred in $48 \%$ of patients versus deterioration in $32 \%$. With MPR induction improvement was reported in $52 \%$ of patients versus in $28 \%$ deterioration. After 1 year of thalidomide maintenance, $54 \%$ of patients improved versus $32 \%$ deteriorated. For lenalidomide maintenance these figures were $61 \%$ versus $19 \%$, respectively. The results for all other subscales are presented in Figure 2. Clinically relevant deterioration in peripheral neuropathy was significantly more frequently reported in the patients treated with MPT-T than in the patients treated with MPR-R, both after induction $(55 \%$ vs. $27 \% ; P<0.001)$ and after maintenance $(63 \%$ vs. $31 \% ; P=0.003)$. A significantly higher percentage of patients treated with MPR reported clinically relevant worsening of diarrhea, compared to MPT, however after induction only $(31 \%$ vs. $9 \% ; P<0.001)$.

\section{Changes in HRQoL within each treatment arm during induction and maintenance}

In both arms, a significant improvement in HRQoL over time was observed for the majority of scales, irrespective of the received treatment. Global QoL, role and emotional functioning, fatigue, pain, and future perspective improved clinically relevant in both arms. In addition, patients who were treated with thalidomide reported a clinically relevant improvement in social functioning, insomnia and appetite loss, while physical functioning improved in patients who were treated with lenalidomide (see Online Supplementary Table S2 for significant changes over time; see Figure 3 and Online Supplementary Figure S2 for clinical meaningful changes within arms). Overall, these HRQoL changes corresponded to medium clinical effects, except for pain reduction, which corresponded to a large clinical effect. ${ }^{24}$ In both arms, pain reduction was observed, irrespective of the number of bone lesions $(0 \mathrm{vs}$. 1-2 vs. $\geq 3$ ) (Online Supplementary Figure S3). In general, clinically meaningful improvement occurred from T3 and T4 onwards only (i.e. after six and 12 months of maintenance therapy). In contrast, global QoL, future perspective and pain improved already during induction therapy and was sustained throughout the whole treatment (Figure 3 and Online Supplementary Figure S2). Patients treated with MPT-T reported a statistically significant, but not clinically meaningful increase (small effect according to Cocks ${ }^{24}$ ) in constipation and side effects of treatment $(P=0.003$ and $P<0.001$ respectively, see Figure 3, Online Supplementary 
Table S2 and Online Supplementary Figure S2). Patients treated with MPR-R reported a statistically significant and clinically meaningful increase (small effect according to Cocks $)^{24}$ in diarrhea $(P<0.001$, see Figure 3 and Online Supplementary Table S2). Peripheral neuropathy worsened in both arms (both $P<0.001$, see Online Supplementary Table S2), being clinically meaningful in patients treated with thalidomide only (Figure 3).

\section{Clinically relevant differences between arms in HRQOL course during induction and maintenance}

During treatment, clinically meaningful differences occurred between arms in 13 of 21 scales (Figure 3 and Online Supplementary Figure S2). Patients treated with MPT-T reported less diarrhea at all follow-up time points (difference between arms was of medium clinical effect, according to $\operatorname{Cocks}^{23}$ ), pain at T1, fatigue at T2, and insomnia and appetite loss at T1 and T4. MPR-R treated patients reported better future perspective, physical and role functioning at $\mathrm{T} 4$, better cognitive functioning at $\mathrm{T} 1$ and $\mathrm{T} 4$, and body image at T3, compared to the patients treated with MPT-T. In addition, patients treated with MPT-T reported more side effects of treatment at T3 and T4 and more constipation and peripheral neuropathy at all follow-up time points. According to the definition of Cocks, all the differences between the arms were of small clinical effect at the largest, except where stated differently (Online Supplementary Table S2). ${ }^{23}$

\section{Changes in HRQOL within and between treatment arms during maintenance only}

We performed an analysis of a subset of 242 patients who started and continued maintenance treatment for at least three months and of whom a T2 questionnaire was available: 95 of 146 patients who started with thalidomide maintenance therapy (65\%) and 147 of 174 patients who started with lenalidomide maintenance therapy (84\%). At the start of maintenance, there was already a significant difference in HRQoL in constipation, side effects of treatment and neuropathy (less in MPR treated patients) and diarrhea (less in MPT treated patients) (Online Supplementary Table S1). During maintenance treatment, a statistically significant reduction in appetite loss was reported in both arms (thalidomide $P=0.003$, lenalidomide $P<0.001)$. In addition, during lenalidomide maintenance, a
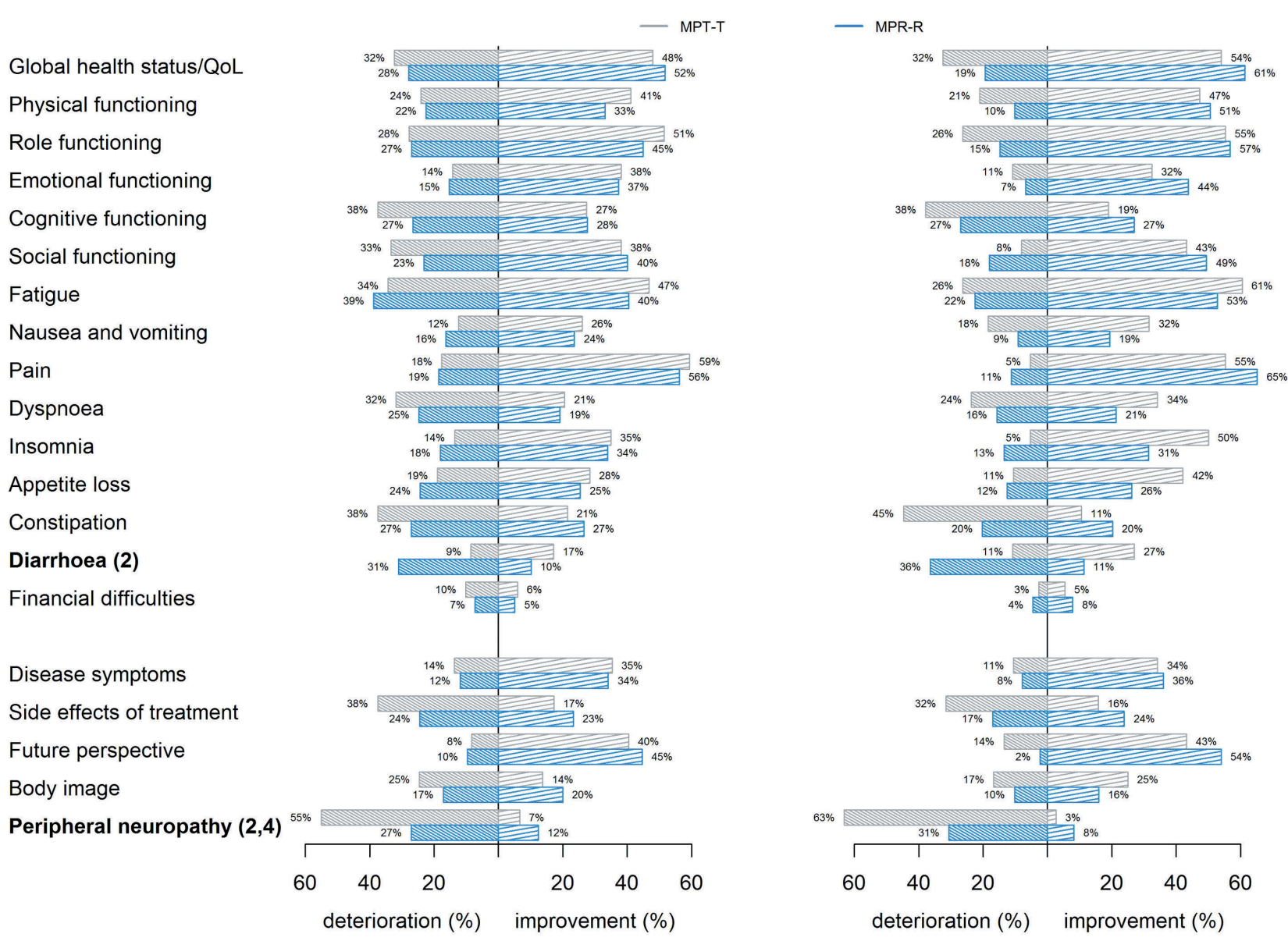

Figure 2. Responders. The percentage of patients reaching a clinically relevant change in health-related quality of life (HRQoL), e.g. reaching the minimal important difference (MID) threshold for within group change during the induction phase (T2) and induction and maintenance phase together (T4). A significant difference between the arms with respect to the percentage of patients improving or deteriorating by more than the MID was observed for diarrhoea and peripheral neuropathy at $\mathrm{T} 2$ and for peripheral neuropathy at T4. 
significant improvement was observed in global QoL $(P=0.003$, clinically relevant at T3), physical- $(P<0.001)$ and role functioning $(P<0.001$, clinically relevant at $\mathrm{T} 4)$, fatigue $(P<0.001)$ and dyspnea $(P=0.004)$. In contrast, no significant improvement occurred during thalidomide maintenance. There was even statistically significant worsening of peripheral neuropathy symptoms $(P<0.001$, clinically relevant at both T3 and T4) (Online Supplementary Table S3 and Online Supplementary Figure S4). Between arms, there were clinically meaningful differences in physical and role functioning (better with lenalidomide), in appetite loss (worse with lenalidomide) and in neuropathy (worse with thalidomide) (Online Supplementary Table S4 and Online Supplementary Figure S4). All differences in OLQ-C30 scales were of small effect size (of note for neuropathy, no effect sizes are available). ${ }^{23}$

\section{Analyses to account for missing data due to different discontinuation rates between arms}

Because more patients in the thalidomide arm discontinued treatment compared to the lenalidomide $\mathrm{arm}^{17}$, HRQoL of patients on protocol in the thalidomide arm
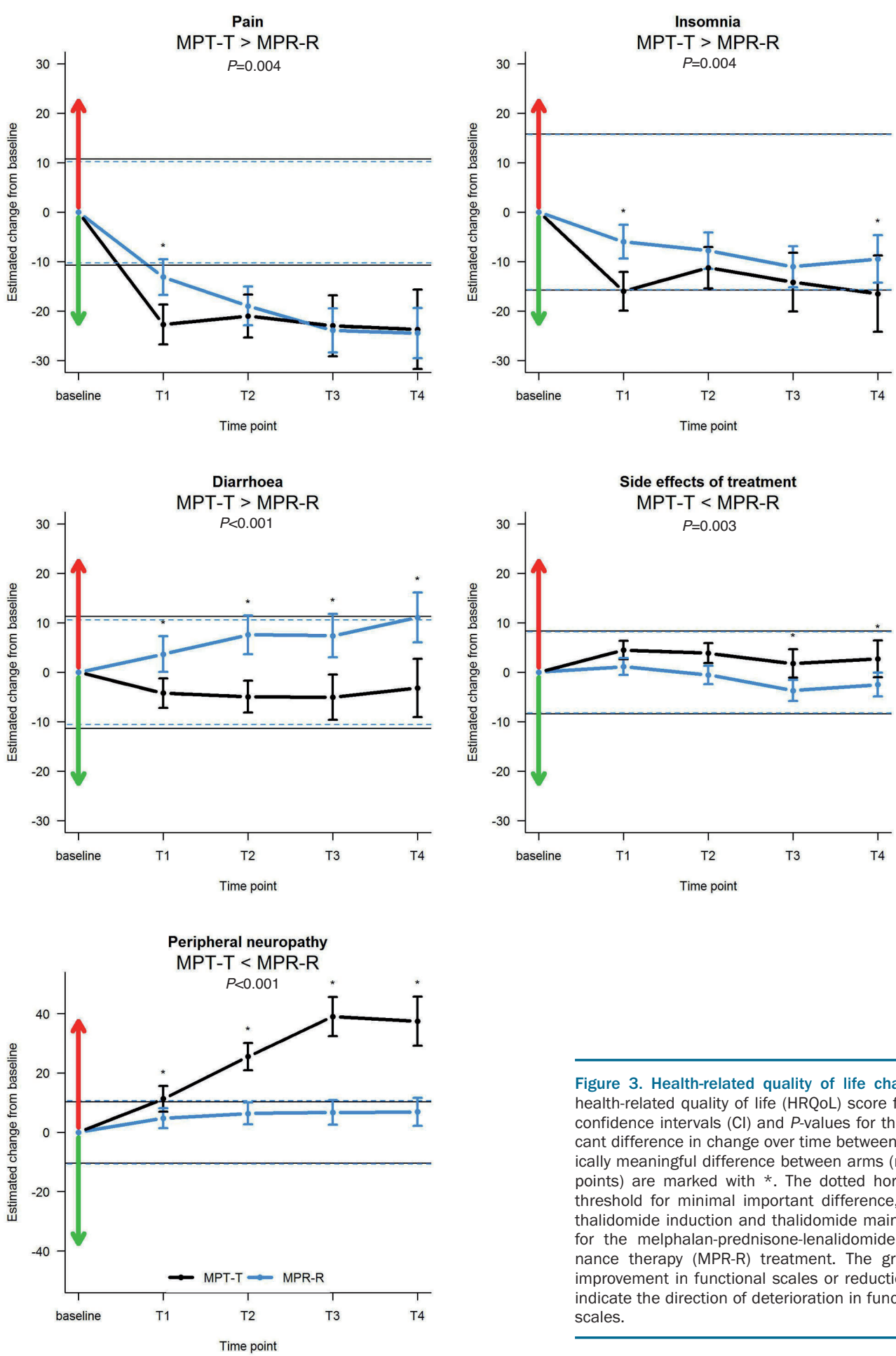

Figure 3. Health-related quality of life change over time. Estimated change in health-related quality of life (HRQOL) score from baseline with corresponding 95\% confidence intervals $(\mathrm{Cl})$ and $P$-values for the five scales with a statistically significant difference in change over time between treatment arms. Time points with clinically meaningful difference between arms (minimal important difference [MID] $>5$ points) are marked with *. The dotted horizontal line represents the calculated threshold for minimal important difference, the black for melphalan-prednisonethalidomide induction and thalidomide maintenance therapy (MPT-T) and the blue for the melphalan-prednisone-lenalidomide induction and lenalidomide maintenance therapy (MPR-R) treatment. The green arrows indicate the direction of improvement in functional scales or reduction in symptom scales. The red arrows indicate the direction of deterioration in functional scales or worsening of symptom scales. 
could have been overestimated and toxicities underestimated. We performed additional analyses to exclude such a potential bias. Although it was not required, a questionnaire was available after treatment discontinuation ("off protocol" questionnaires) from 90 patients (53 MPT-T and 37 MPR-R), of which 84 could be matched with questionnaires from patients on protocol with comparable age, $\mathrm{WHO}$, disease status, treatment arm and period. The global QoL for patients on protocol was comparable to patients off protocol (mean score 59.9 vs. 66.3 points, respectively, $P=0.043$; Online Supplementary Table S5). Secondly, in general, the HRQoL course did not differ between patients who discontinued therapy early ( $\leq \mathrm{T} 2$, e.g. during induction) and those who discontinued therapy late or never (>T2, e.g. from start maintenance; Online Supplementary Table S6 and Online Supplementary Figure S6). Lastly, global QoL over time did not differ between patients discontinuing treatment due to investigatorreported peripheral neuropathy and patients continuing treatment until 12 months of maintenance therapy $(P<0.001$; Online Supplementary Table S7 and Online Supplementary Figure S6). These analyses support the absence of bias in our analyses.

\section{Effect modification of HRQOL change by baseline WHO} performance, gender, age and treatment response

Baseline WHO performance status appeared to be an effect modifier of HRQoL change during treatment for 7 of 21 scales in MPT-T treated- and in 12 of 21 scales in MPR-R treated patients (Figure 4 for global QoL and Online Supplementary Table S8 and Online Supplementary Figure S8 for other subscales). A low performance status (WHO score $\geq 2$ ) was associated with a statistically significant overall lower HRQoL at baseline (data not shown), which became comparable to the HROoL of patients with

Global health status/QoL: MPT-T

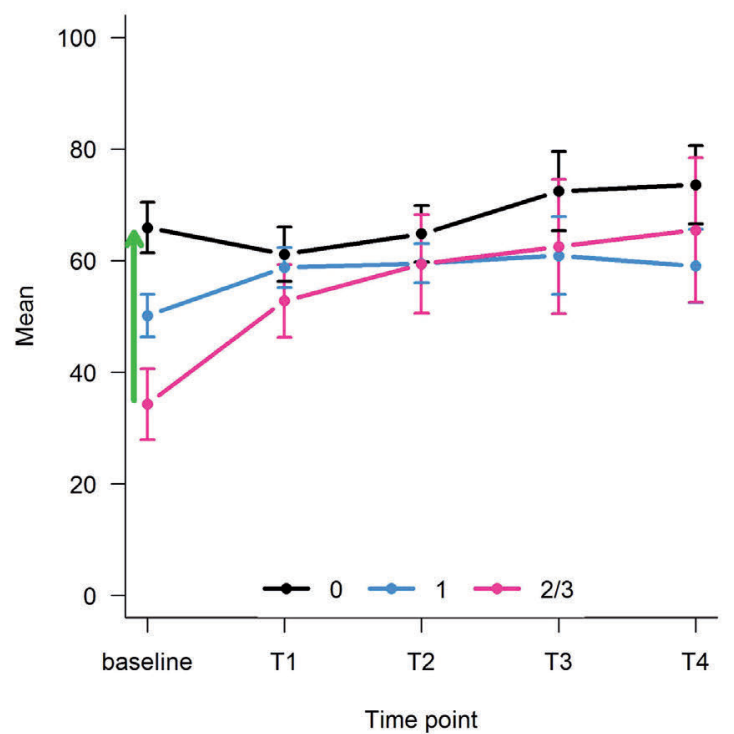

baseline WHO performance status 0-1 during treatment (Online Supplementary Table S8 and Online Supplementary Figure S7). Sex, age ( $\leq 75$ years vs. $>75$ years) and treatment response ( $\geq$ partial response $v s$. stable/progressive disease) did not modify HRQoL course (data not shown). The only exception was observed for patients treated with MPT-T $\leq 75$ years of age, who experienced more peripheral neuropathy during treatment compared to those $>75$ years (Online Supplementary Table 59 and Online Supplementary Figure S8).

\section{Patient- versus investigator-reported peripheral neuropathy}

Figure 5 shows the patient-reported peripheral neuropathy, as described in the method section, in comparison to the investigator-reported CTCAE score at the given time point. After dichotomizing peripheral neuropathy to "no" or "mild" peripheral neuropathy versus "moderate" or "severe neuropathy", the kappa between patient-reported and investigator-reported peripheral neuropathy was 0.33 (95\% CI: $0.29-0.36)$. In 213 of 1,599 (13.3\%) evaluable questionnaires a discordance was found between neuropathy grades reported by the patients versus investigators. In $76 \%$ of the cases, the investigator interpreted the grade of neuropathy lower than experienced by the patient.

\section{Discussion}

IMiD-based first line treatment of elderly non-transplant eligible patients with NDMM improves both progression free and overall survival. ${ }^{25-28}$ We here show that patients also perceive clinically relevant HRQoL benefits of induction therapy with thalidomide and lenalidomide

igure 4. Effect modification of global quality of life by World Health Organisation status. Mean global quality of life (QoL) course over time with corresponding $95 \%$ confidence intervals $(\mathrm{Cl})$ for each time point for $(\mathrm{A})$ melphalan-prednisone-thalidomide induction and thalidomide maintenance therapy (MPT-T) and (B) patients treated with melphalan-prednisone-lenalidomide induction and lenalidomide maintenance therapy (MPR-R), differentiated by baseline World health organisation (WHO) performance status 0 versus 1 versus 2/3. The black curve represents patients with baseline WHO status 0 , the blue curve the patients with WHO status 1 and the pink curve the patients with WHO status $2 / 3$. The green arrows indicate the direction of improvement in functional scales and reduction in symptom scales. 
followed by maintenance therapy. Importantly, there was a clinically relevant decrease in pain, defined as large by Cocks et al., in both arms. Clinically meaningful diarrhea developed in approximately $30 \%$ of patients during lenalidomide treatment, being of medium clinical effect and significantly higher than in patients treated with thalidomide. Importantly, in patients who reached maintenance, there was no clinically relevant further deterioration of diarrhea during MPR-R therapy. In contrast, clinically meaningful peripheral neuropathy developed in approximately $60 \%$ of patients during thalidomide treatment, being significantly higher than in lenalidomidetreated patients, both during induction and maintenance.

In general, the improvement in HRQoL subscales reached clinical relevance after six and 12 months of maintenance therapy only, with the exception of global QoL, future perspective and pain, which improved early during induction therapy and sustained during treatment. A subanalysis of patients who started maintenance therapy and were treated for at least three months, showed that lenalidomide resulted in a clinically meaningful improvement in global QoL and role functioning over time, without any clinically meaningful deteriorations. In contrast, there was no clinical benefit of thalidomide maintenance treatment, only clinically relevant worsening of peripheral neuropathy occurred.

Importantly, patients with a poor WHO performance status of $\geq 2$ at baseline reached similar HRQoL during treatment, compared to patients with a better WHO performance status at baseline, irrespective of the treatment arm. Although this could be explained by regression to the mean, indicating that the most pronounced improvement can be achieved in patients with the worst HRQoL, it is reasonable to suppose that the performance status can be negatively affected by the disease and therefore that treatment also improves HRQoL of the physically most compromised patients. Therefore, treatment should not be automatically withheld from those patients.

Our findings are in line with previous data on HRQoL describing improvement in QoL during treatment. ${ }^{6}$ In addition, we provide data about HRQoL during up to 1 year maintenance therapy with lenalidomide and thalidomide, being rarely reported in the current literature. ${ }^{10-13,16}$ In view of the continuous lenalidomide treatment approaches, our data showing clinically meaningful improvement of global QoL during maintenance therapy is important for clinical practice. In the FIRST trial, no such improvement was reported; the global QoL remained stable, which might have been attributed to the higher dose of lenalidomide and the continuation of dexamethasone, known to be associated with side effects that can hamper QoL. . In the MM-015 trial, a statistically significant, but not clinically relevant deterioration in global QoL after six months of maintenance therapy was observed. ${ }^{11}$ This cannot be accounted for by a difference in the percentage of patients starting maintenance, the dose of lenalidomide, response rates or different QoL evaluation methods. The fact that thalidomide maintenance has only limited impact on global QoL is in line with the data of the HOVON 49 and the MRC IX data. The latter even show an inferior global QoL at three months with a trend to further detriment during maintenance treatment. ${ }^{8,12}$

A limitation of our and HRQoL studies of patients with $\mathrm{MM}$ in general, is the fact that firstly, long term data reflect a subset of patients who tolerate remaining in treat- ment. Secondly, we collected no data after discontinuation of the study although such results would rather reflect the outcome of subsequent therapies. ${ }^{29}$ Therefore, biases are common, and comparisons between therapies with different toxicities and discontinuation rates are difficult. Especially, as missing data are not missing at random when related to toxicity and cannot be corrected for. ${ }^{30}$ Such bias might be present in our sub-analyses of patients starting maintenance therapy, showing benefit for patients treated with lenalidomide only. Therefore, this can only be concluded for those patients who did benefit from MPR induction and were able to continue lenalidomide. In addition, the low number of patients continuing thalidomide might be the cause of a lack of finding statistically significant changes in their HROoL during maintenance.

Interestingly, the higher incidence of peripheral neuropathy with thalidomide, both clinically meaningful to patients and according to CTCAE reported by physicians, did not translate into an inferior global QoL, neither in our study nor the FIRST trial. ${ }^{9}$ Via several analyses, we excluded a bias in global QoL evaluation due to missing HRQoL questionnaires from patients who discontinued treatment because of peripheral neuropathy. The fact that peripheral neuropathy did not negatively affect HRQoL was surprising. The opposite has been reported. ${ }^{31}$ Our observation might be explained by response shift, which is a wellknown phenomenon in longitudinal QoL research. It reflects the probability that a patient's standards and values change over time. ${ }^{32}$ Patients with MM might adapt to their worsening function and increased symptoms and thereby not allow these aspects to affect their global QoL. ${ }^{33}$ However, it cannot be excluded that the global QoL scale of the EORTC OLQ-C30 questionnaire has limitations in detecting the negative impact of toxicity on HRQoL. This discussion was addressed in a meta-analysis by Schuurhuizen and colleagues, ${ }^{34}$ who also reported sim-

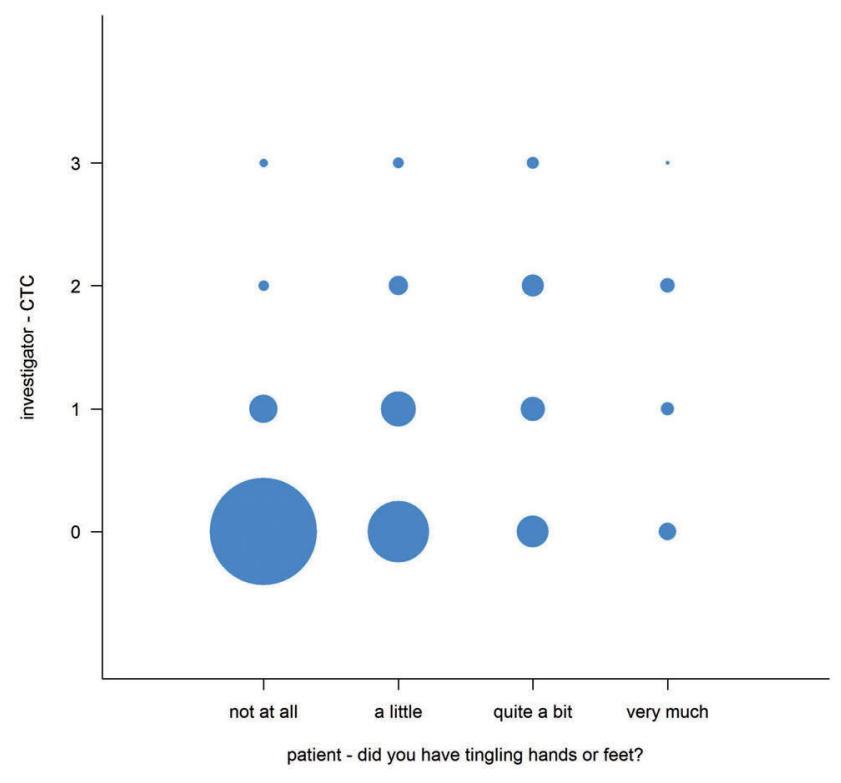

Figure 5. Patient- versus investigator-reported peripheral neuropathy. Patientreported peripheral neuropathy compared to investigator-reported peripheral neuropaty assessed by National Cancer Institute Common Terminology Criteria for Adverse Events (CTCAE) version 3.0. The surface of the circles reflects the absolute number of patients plus investigators. 
ilar global QoL in experimental versus control arms in patients with colorectal cancer, despite higher toxicity rates in the former. The authors question whether the global QoL assessment, using the very brief two-item global QoL scale, has sufficient measurement precision to detect a difference in global QoL over time. However, this has been challenged by others stating that QoL is a complex and multidimensional concept, not caused by side effects only ${ }^{35}$ Importantly, both those in favor and those against the limitations of the global QoL scale indicate the need for development for more sensitive patient-reported QoL instruments. ${ }^{36}$ This is also supported by our data on peripheral neuropathy. We defined 'tingling' of hands or feet as peripheral neuropathy. Although this approach is not validated, we found a discrepancy between patient and physician-reported neuropathy in 13\% of questionnaires, the majority $(76 \%)$ being explained by lower reporting by physicians, which has been reported previously, both for neuropathy and other symptoms. ${ }^{37-39}$ The use of the EORTC OLO-CIPN20 questionnaire might improve concordance between patients and physicians. ${ }^{40}$ The need for flexible and tailored patient reported outcome (PRO) measurements has recently also been advocated by Thanarajasingam and colleagues and these have been developed by the EORTC ${ }^{36,41}$ Furthermore, the PRO version of the Common Terminologies Criteria for Adverse Events (PRO-CTCAE) for self-reported toxicities developed by the National Institute of Health is another valuable tool. ${ }^{42,43}$

In conclusion, we found that the treatment with MPT-T and MPR-R improved HRQoL in elderly patients with NDMM and in general is clinically meaningful to the patients during maintenance therapy only. This supports the current paradigm of continuous treatment, not only improving survival, but also maintaining, and even improving, specific subscales of HRQoL. Although clinically relevant diarrhea developed in patients treated with lenalidomide, this did not negatively affect the global QoL during induction and maintenance. Moreover, currently it is known that bile acid malabsorption plays an important role, which can be treated with bile acid sequestrants. ${ }^{44}$ Clinically significant peripheral neuropathy precluded long term thalidomide treatment in the majority of patients and appeared not to improve HRQoL in those patients who continued therapy.

\section{Funding}

Dutch Cancer Society KWF VU-2008 4246, Celgene.

\section{References}

1. Rajkumar SV, Dimopoulos MA, Palumbo A, et al. International Myeloma Working Group updated criteria for the diagnosis of multiple myeloma. Lancet Oncol. 2014;15(12):e538-548.

2. Kyle RA, Rajkumar SV. Multiple myeloma. Blood. 2008;111(6):2962-2972.

3. Jordan K, Proskorovsky I, Lewis P, et al. Effect of general symptom level, specific adverse events, treatment patterns, and patient characteristics on health-related quality of life in patients with multiple myeloma: results of a European, multicenter cohort study. Support Care Cancer. 2014; 22(2):417-426.

4. Johnsen AT, Tholstrup D, Petersen MA, Pedersen L, Groenvold M. Health related quality of life in a nationally representative sample of haematological patients. Eur J Haematol. 2009:83(2):139-148.

5. Priscilla D, Hamidin A, Azhar MZ, Noorjan KON, Salmiah MS, Bahariah K. Quality of life among patients with hematological cancer in a Malaysian hospital. Med J Malaysia. 2011;66(2):117-120.

6. Nielsen LK, Jarden M, Andersen CL, Frederiksen H, Abildgaard N. A systematic review of health-related quality of life in longitudinal studies of myeloma patients. Eur J Haematol. 2017;99(1):3-17

7. Waage A, Gimsing P, Fayers $P$, et al. Melphalan and prednisone plus thalidomide or placebo in elderly patients with multiple myeloma. Blood. 2010;116(9):1405-1412.

8. Verelst SG, Termorshuizen F, Uyl-de Groot $\mathrm{CA}$, et al. Effect of thalidomide with melphalan and prednisone on health-related quality of life (HROoL) in elderly patients with newly diagnosed multiple myeloma: a prospective analysis in a randomized trial. Ann Hematol. 2011:90(12):1427-1439.

9. Delforge M, Minuk L, Eisenmann JC, et al. Health-related quality-of-life in patients with newly diagnosed multiple myeloma in the FIRST trial: lenalidomide plus low-dose dexamethasone versus melphalan, prednisone, thalidomide. Haematologica. 2015; 100(6):826-833

10. Stewart AK. Melphalan, prednisone, and thalidomide vs melphalan, prednisone, and lenalidomide (ECOG E1A06) in untreated multiple myeloma. Blood. 2015;126(11): 1294-1301.

11. Dimopoulos MA, Delforge M, Hajek R, et al. Lenalidomide, melphalan, and prednisone, followed by lenalidomide maintenance, improves health-related quality of life in newly diagnosed multiple myeloma patients aged 65 years or older: results of a randomized phase III trial. Haematologica. 2013;98(5):784-788

12. Royle KL, Gregory WM, Cairns DA, et al. Quality of life during and following sequential treatment of previously untreated patients with multiple myeloma: findings of the Medical Research Council Myeloma IX randomised study. $\mathrm{Br} J$ Haematol. 2018;182(6):816-829.

13. Vogl DT, Delforge M, Song K, et al. Longterm health-related quality of life in transplant-ineligible patients with newly diagnosed multiple myeloma receiving lenalidomide and dexamethasone. Leuk Lymphoma. 2018;59(2):398-405

14. Benboubker L, Dimopoulos MA, Dispenzieri A, et al. Lenalidomide and dexamethasone in transplant-ineligible patients with myeloma. N Engl J Med. 2014; 371(10):906-917

15. Dimopoulos MA, Palumbo A, Hajek R, et al. Factors that influence health-related quality of life in newly diagnosed patients with multiple myeloma aged $>65$ years treated with melphalan, prednisone and lenalidomide followed by lenalidomide maintenance: Results of a randomized trial. Leuk Lymphoma. 2014;55(7):1489-1497.

16. Abonour R, Wagner L, Durie BGM, et al.
Impact of post-transplantation maintenance therapy on health-related quality of life in patients with multiple myeloma: data from the Connect(R) MM Registry. Ann Hematol. 2018:97(12):2425-2436.

17. Zweegman S, van der Holt B, Mellqvist UH, et al. Melphalan, prednisone, and lenalidomide versus melphalan, prednisone, and thalidomide in untreated multiple myeloma. Blood. 2016;127(9):1109-1116.

18. Cocks K, Cohen D, Wisløff F, et al. An international field study of the reliability and validity of a disease-specific questionnaire module (the OLO-MY20) in assessing the quality of life of patients with multiple myeloma. Eur J Cancer. 2007;43(11):16701678.

19. Aaronson NK, Ahmedzai S, Bergman B, et al. The European Organization for Research and Treatment of Cancer OLO-C30: a quality-of-life instrument for use in international clinical trials in oncology. J Natl Cancer Inst. 1993;85(5):365-376

20. Giesinger JM, Kieffer JM, Fayers PM, et al. Replication and validation of higher order models demonstrated that a summary score for the EORTC OLQ-C30 is robust. J Clin Epidemiol. 2016;69:79-88.

21. Fayers $\mathrm{P}$, Aaronson NK, Biordal $\mathrm{K}$, Groenvold M, Curran D, Bottomley A, on behalf of the EORTC Quality of Life Group. The EORTC OLQ-C30 Scoring Manual (3rd Edition). European Organisation for Research and Treatment of Canceer, Brussels 2001.

22. King MT. A point of minimal important difference (MID): a critique of terminology and methods. Expert Rev Pharmacoecon Outcomes Res. 2011;11(2):171-184

23. Cocks K, King MT, Velikova G, Martyn StJames M, Fayers PM, Brown JM. Evidencebased guidelines for determination of sample size and interpretation of the European Organisation for the Research and Treatment of Cancer Quality of Life 
